\title{
ESTANDARIZACIÓN DE LA TÉCNICA DE AFLPs PARA LA CARACTERIZACIÓN DE GERMOPLASMA DE CAÑA FLECHA (Gynerium sagittatum Aubl.)
}

\section{AFLPS TECHNIQUE STANDARIZATION FOR ARROW CANE (Gynerium sagittatum Aubl.) GERMPLASM CHARACTERIZATION}

\author{
Hernando H. Rivera ${ }^{1}$, Isidro E. Suárez ${ }^{1}$, Juan D. Palacio ${ }^{2}$ \\ Recibido para evaluación: Septiembre 13 de 2008 - Aceptado para publicación: Mayo 29 de 2009
}

\section{RESUMEN}

La caña flecha (Gynerium sagittatum (Aubl.), especie originaria de la India, constituye la materia prima esencial para la elaboración de artesanías típicas en la Costa Norte Colombiana. Conocer la variabilidad genética de esta especie permite diseñar e implementar estrategias de mejoramiento y conservación de los recursos genéticos. El objetivo del estudio fue desarrollar un protocolo para caracterizar 25 accesiones de caña flecha con AFLPs. El análisis permitió detectar 223 loci que se extendieron desde 43 (E-ACC/M-CAG) hasta 72 loci (E-ACA/M-CTA) con un promedio de 55,75 loci por combinación de iniciadores. La combinación de iniciadores E-ACC/M-CAG generó el mayor porcentaje de polimorfismo (39,53\%). Los diferentes perfiles de locus para todos los individuos indicaron un buen escrutinio del genoma para el análisis de la variabilidad y la estructura genética de la especie. El presente protocolo muestra por primera vez la caracterización genética a nivel molecular en caña flecha.

Palabras clave: AFLP, Gyneryum sagittatum Aubl, caña flecha, variabilidad genética.

\section{ABSTRACT}

The arrow cane (Gynerium sagittatum Aubl.) is the raw material used for typical craftsmanship in the Colombian Northen Coast. Knowing the genetic variability allows to design and develop breeding strategies and genetic plant resource conservation. The objective of the study was to develop an AFLPs protocol to evaluate the genetic variability among 25 arrow cane accessions planted at the Universidad de Córdoba collection. A total of 223 loci ranging from 43 (E-ACC/M-CAG) to 72 loci (E-ACA/M-CTA). Primer average loci was 55,75. Combination E-ACC/M-CAG generated the highest polymorphism $(39,53 \%)$. Locus profiles were different for all individuals in the population indicating a good genome scrutiny for analyzing variability and genetic structure of the species.

Key words: AFLP, Gynerium sagittatum (Aubl. ), arrow cane, genetic variability.

\footnotetext{
${ }^{1}$ Universidad de Córdoba, departamento de Ingeniería Agronómica y Desarrollo Rural. Cra. 6 №. 76-103, Montería-Colombia. Tel: (4) 7908023,Fax: (4) 7860255 - E-mail:isuarez@sinu.unicordoba.edu.co

${ }^{2}$ Instituto de Investigación de Recursos Biológicos "Alexander Von Humboldt". Calle 28A No. 15-09, Bogotá.
} 


\section{INTRODUCCIÓN}

La caña flecha (Gynerium sagittatum Aubl.) es la fuente de fibra para la elaboración de las más tradicionales y populares artesanías colombianas como lo es el sombrero vueltiao. Esta especie se encuentra distribuida en gran parte de la geografía nacional, localizándose espontáneamente a lo largo de riberas de los ríos y zonas de altas precipitaciones. En el marco del programa de investigación número CIUCFCA002-2004 conducente a valorar la riqueza genética de la especie, una colección de genotipos de caña flecha ubicada en la Universidad de Córdoba ha sido caracterizada mediante la descripción de características morfoagronomicas de interés directo para los usuarios (Aramendiz et al., 2005). Aunque de gran importancia agronómica, este método de caracterización tiene ciertas limitantes como son características altamente heredables, frecuentemente muestra poca variación y la expresión de las características esta sujeta a variación ambiental que puede ser difícil de medir. El uso de técnicas moleculares que permiten analizar el polimorfismo directamente al nivel del ADN supera estas limitantes al no estar influenciada por los factores anteriormente anotados (Chawla, 2002); no obstante, debe enfatizarse que debido a su aplicación desde el punto de vista agronómico, la caracterización morfológica no es factible de ser remplazada por las técnicas moleculares, sino mas bien ambos deben ser considerados como complementarios en la caracterización genética (Karpa et al., 1997).

La caracterización molecular mediante la técnica de Polimorfismo de la Longitud de los Fragmentos Amplificados (AFLP) es utilizada frecuentemente como una herramienta para estimar variabilidad genética en una amplia gama de organismos (Bonin et al., 2007). Esta técnica se basa en la digestión del ADN genómico con enzimas de restricción y la posterior amplificación de los fragmentos después de habérsele adicionado adaptadores a los extremos que los hacen complementarios a iniciadores. En la presente investigación se desarrolló un protocolo para caracterizar molecularmente 25 accesiones usando AFLPs con el fin de determinar la diversidad genética existente en la colección.

\section{MATERIALES Y METODOS}

\section{Extracción de ADN}

El material vegetal para la extracción del ADN se obtuvo de plantas adultas establecidas en la Colección de Germoplasma de Caña Flecha ubicada en la Granja Experimental de la Facultad de Ciencias Agrícolas de la Universidad de Córdoba. Los individuos evaluados procedieron de los departamentos de Antioquia, Córdoba, Caldas, Santander, Norte de Santander y Valle el Cauca. La extracción de ADN se realizó de acuerdo con las recomendaciones del fabricante del kit (Qiagen, 2004) con algunas modificaciones. Aproximadamente $20 \mathrm{mg}$ de tejido foliar fueron macerados con nitrógeno líquido. Posteriormente, se adicionaron $400 \mu \mathrm{ldel}$ solución reguladora AP1, $4 \mu \mathrm{l}$ de RNasa A, la mezcla fue incubada a $65^{\circ} \mathrm{C}$ por 10 minutos con agitación cada dos minutos. Seguidamente se adicionaron $130 \mu \mathrm{l}$ de la solución reguladora AP2, con cinco minutos de incubación en hielo seguido por centrifugación durante cinco minutos a 14000 rpm. El sobrenadante fue colectado, transferido a una columna de separación y centrifugado durante dos minutos a $14000 \mathrm{rpm}$. El líquido filtrado fue adicionado con 1,5 volúmenes de solución reguladora AP3/E. Aproximadamente $650 \mu \mathrm{l}$ de la mezcla fueron transferidos a una columna de separación y centrifugado durante 1 minuto a $8000 \mathrm{rpm}$ descartando el líquido filtrado; este paso fue repetido una vez. La columna con $50 \mu$ l de solución buffer fue incubada por 5 minutos a temperatura ambiente $y$ centrifugado durante 1 minuto a 8000 rpm, este paso se repitió una vez, y la muestra obtenida fue almacenada $\mathrm{a}-80^{\circ} \mathrm{C}$.

\section{Desarrollo de AFLPs y análisis de datos}

La metodología para la generación de los AFLPs se basó en el protocolo descrito por Vos et al. (1995). El ADN genómico fue digerido con EcoRI y MseI y posteriormente dos adaptadores fueron adicionados a los extremos de los fragmentos, los cuales fueron amplificados usando PCR (Polymerase Chain Reaction). Las amplificaciones fueron conducidas en un volumen final de $20 \mu \mathrm{l}$ por muestra, conteniendo $10 \mu \mathrm{m}$ de cada primer $25 \mathrm{mM}$ de cada dNTPs, $8.1 \mu \mathrm{l}$ de agua destilada, 2 $\mu \mathrm{l}$ de buffer plus Mg de PCR 10X, 0,2 $\mu \mathrm{l}$ de Taq 
ADN polimerasa, y $5 \mu \mathrm{l}$ de ADN. Los ciclos de PCR fueron: $94^{\circ} \mathrm{C}$ por 30 segundos, $65^{\circ} \mathrm{C}$ por 30 segundos y $72{ }^{\circ} \mathrm{C}$ por 60 segundos, con un total de 23 ciclos. De los resultados obtenidos a partir de las ocho combinaciones de iniciadores probadas, se realizó un análisis con el fin de encontrar las combinaciones de iniciadores que permitiera diferenciar el mayor número de loci polimórficos entre las diferentes muestras. Los productos de AFLP se separaron con electroforesis vertical en geles de acrilamida al $6 \%$. El gel fue teñido con nitrato de plata y posteriormente revelado de acuerdo con los protocolos descritos por Bassam (1991).

La presencia (valor $=1$ ) o ausencia $($ valor $=0$ ) de bandas específicas originadas en la amplificación de los fragmentos generados por la digestión se transformaron en una matriz binaria. Se calculó una segunda matriz de similaridad utilizando el paquete estadístico GELSTATS y un dendrograma que permitió agrupar las diferentes variantes encontradas. La diversidad genética se estimó con el índice de Dice (1945), adaptado por Nei y Li (1979) para datos moleculares.

El índice promedio de los valores de similitud por par de individuos se calculó mediante la ecuación: $\mathrm{Sij}=2 \mathrm{a} /(2 \mathrm{a}+\mathrm{b}+\mathrm{c}) ;$ donde: $\mathrm{Sij}$ es la similitud entre los individuos $i$ y $j$, a es el número de loci compartidos por $\mathrm{i}$ y $j$, b es el número de loci presentes en i pero ausentes en $j$, y c es el número de loci presentes en $\mathrm{j}$ pero ausentes en i. Las matrices y los dendrogramas de similitud se calcularon con el programa NTSYS-PC, versión 2.02i mediante el método UPGMA y el agrupamiento SAHN (Rohlf, 1998), respectivamente. Para el análisis de los datos de AFLP se estudió las relaciones entre individuos mediante el análisis de correspondencia múltiple (ACM) con toda la población para obtener una representación grafica de la estructura o relación de parentesco de las introducciones.

\section{RESULTADOS Y DISCUSIÓN}

\section{Desarrollo de AFLPs y análisis de datos}

EI ADN obtenido se observó libre de impurezas y en condiciones de poca degradación, apto para servir como templete para el análisis (Figura A). Los fragmentos de ADN amplificados estuvieron en un rango entre 20 y 330 pb de acuerdo con las bandas observadas en el gel resuelto (Figura B). Los datos colectados a partir de las cuatro combinaciones de iniciadores seleccionadas por ser las mas polimórficas permitieron detectar polimorfismos que oscilaron entre 25,8 y $39,53 \%$, siendo la combinación de inciadores EACC/M-CAG la que generó el mayor porcentaje de polimorfismo y E-ACT/M-CAA la menos polimórfica de las cuatro seleccionadas (Tabla 1). Los mismos resultados muestran que las

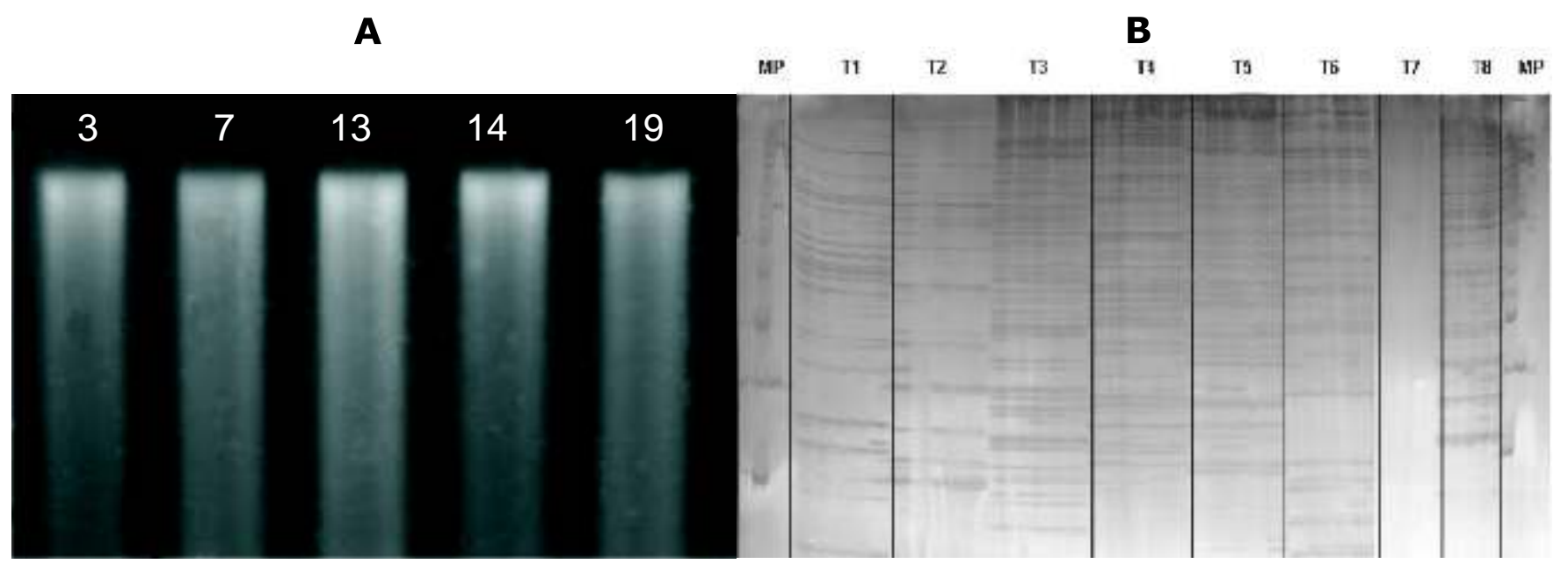

Figura 1. A: ADN de las accesiones $3,7.13,14$ y 19 de caña flecha digerido con enzimas EcoRI y MseI. B. Fragmentos de ADN de caña flecha amplificados a partir de ocho combinaciones de primers. 
amplificaciones realizadas generaron un total de 223 loci, observándose que las combinaciones de iniciadores más numerosas en loci, generaron el menor grado de polimorfismo, pero de forma inversa las que generaron el menor número de bandas resultaron en el mayor número de loci monomórficos (Tabla 1).

Empleando algunas de las mismas combinaciones de iniciadores utilizadas en el presente estudio, Selvi et al. (2005) evaluó la diversidad genética en poblaciones de caña de azúcar encontrando un total de 989 loci con un promedio de 82,4 loci por combinación, y rangos entre 54 (E-ACA/M-CTA) y 109 (E-ACC/M-CTA) loci. Lima et al. (2002) utilizando AFLPs para estudiar la variabilidad genética en una colección de 79 cultivares brasileños de caña de azúcar encontró 2331 loci de los cuales 1121 fueron polimórficos originando un índice promedio de polimorfismo del $48 \%$. Previamente, Besse et al. (1998) en otro estudio sobre similaridad entre genotipos de caña de azúcar reportó resultados igualmente concordantes con los reportados en caña de azúcar en los dos estudios anteriores y los encontrados en el presente estudio, lo cual puede interpretarse como un signo de reproducibilidad de la técnica y su potencial para el estudio de diversidad genética en caña flecha.

La técnica de AFLP ha demostrado que pueden generar estimaciones confiables en estudios de diversidad genética en distintas especies vegetales mostrando altos niveles de diferenciación genética, como en cultivares de Saccharum spp (Lima et at., 2002; Selvi et al., 2005), Oryza sativa L. (Prashanth et al., 2002), Manihot esculenta (Fregene et al., 2000). La información molecular de diversidad y distancia genética para determinar variabilidad en colecciones ha sido también utilizada en diversos trabajos de investigación en programas de mejoramiento, así como para evaluación de redundancia y deficiencia de los bancos de germoplasma (Phillips et al., 1993; Newbury y Ford-Lloyd, 1993), en tomate (Young et al., 1988), brassica (Ferreira et al., 1995) y maíz (Bentolila et al., 1991).

Ningunas de las 25 muestras tomadas de las accesiones revelaron un perfil idéntico de ADN, mostrando un fingerprint único para cada genotipo, lo cual indica un nivel alto de diferenciación determinado por los marcadores de AFLP y ausencia de duplicados genéticos en las muestras analizadas. Siendo la caña flecha una especie que es propagada específicamente por métodos asexuales de reproducción, podría esperarse que existiera poca variabilidad genética en los individuos muestreados a pesar de su distancia geográfica; sin embargo, al analizar los resultados generados por las cuatro combinaciones de iniciadores se observa que hay un porcentaje promedio relativamente alto de

Tabla 1. Grado del polimorfismo detectado en los cultivares de caña flecha con las diversas combinaciones de iniciadores utilizadas.

\begin{tabular}{ccccc}
\hline $\begin{array}{c}\text { Combinación de } \\
\text { primers }\end{array}$ & \multicolumn{2}{c}{ Número de fragmentos } & & Polimorfismo (\%) $^{\mathbf{2}}$ \\
\cline { 2 - 4 } & Total & $\mathbf{P}$ & $\mathbf{M}$ & \\
\hline E-ACC/M-CAG & 43 & 17 & 26 & 39.53 \\
E-ACA/M-CAT & 52 & 20 & 32 & 38.46 \\
E-ACA/M-CTA & 72 & 23 & 49 & 31.94 \\
E-ACT/M-CAA & 56 & 15 & 41 & 25.80 \\
Total & 223 & 75 & 148 & $33.63^{3}$ \\
\hline
\end{tabular}

${ }^{1} \mathrm{P}=$ Polimórfico, $\mathrm{M}=$ Monomórfico

${ }^{2}$ Determinado con base en el número de fragmentos polimórficos fuera del número total de fragmentos amplificados por una combinación de cebadores a través de todas las variedades.

${ }^{3}$ Polimorfismo promedio 
polimorfismo $(33,63 \%)$ consecuente con un grado relativamente elevado de variabilidad, proporcionando así un índice alto de confianza en la identidad varietal de la especie.

Los resultados de estos estudios nos permiten afirmar que las combinaciones de iniciadores utilizadas pueden recomendarse para realizar fingerprint y generar estimaciones confiables en estudios de diversidad genética en caña flecha. El dendrograma (Figura 2) muestra la conformación de tres grupos genéticos perfectamente diferenciados con base en la información generada en los AFLPs. Esta diferenciación grupal concuerda con la reportada previamente por Araméndiz et al. (2005) con base en características morfoagronómicas, las cuales permitieron determinar que las accesiones de los grupos uno y tres se caracterizan por presentar textura áspera y abundante pubescencia, mientras que los miembros del segundo grupo genético se caracterizan por presentar textura de la fibra suave y vaina con escasa pubescencia. Balasaravanan et al. (2003) complementando un estudio de caracterización morfológica con la técnica de AFLPs pudo caracterizar 49 cultivares de té (Camellia sinensis , Camellia assamica y Camellia spp) concluyeron que la diversidad genética del banco de germoplasma estudiado era estrecha. Resultados similares obtuvo Ayanog'lu et al. (2007) al utilizar AFLPs para complementar estudios de caracterización morfológica en ciruelo (Prunus cerasifera L.) proporcionando una discriminación más específica que la morfológica para identificar accesiones deseables y potencialmente importantes a nivel económico asociados a la calidad de fruta.

Los resultados obtenidos en el presente trabajo permiten estimar un alto grado de confiabilidad de la técnica de caracterización molecular con AFLPs para evaluar variabilidad genética en poblaciones de caña flecha y tomarse como una base para continuar la búsqueda de nuevos genotipos con el fin de obtener materiales promisorios y aumentar la diversidad genética de la colección.

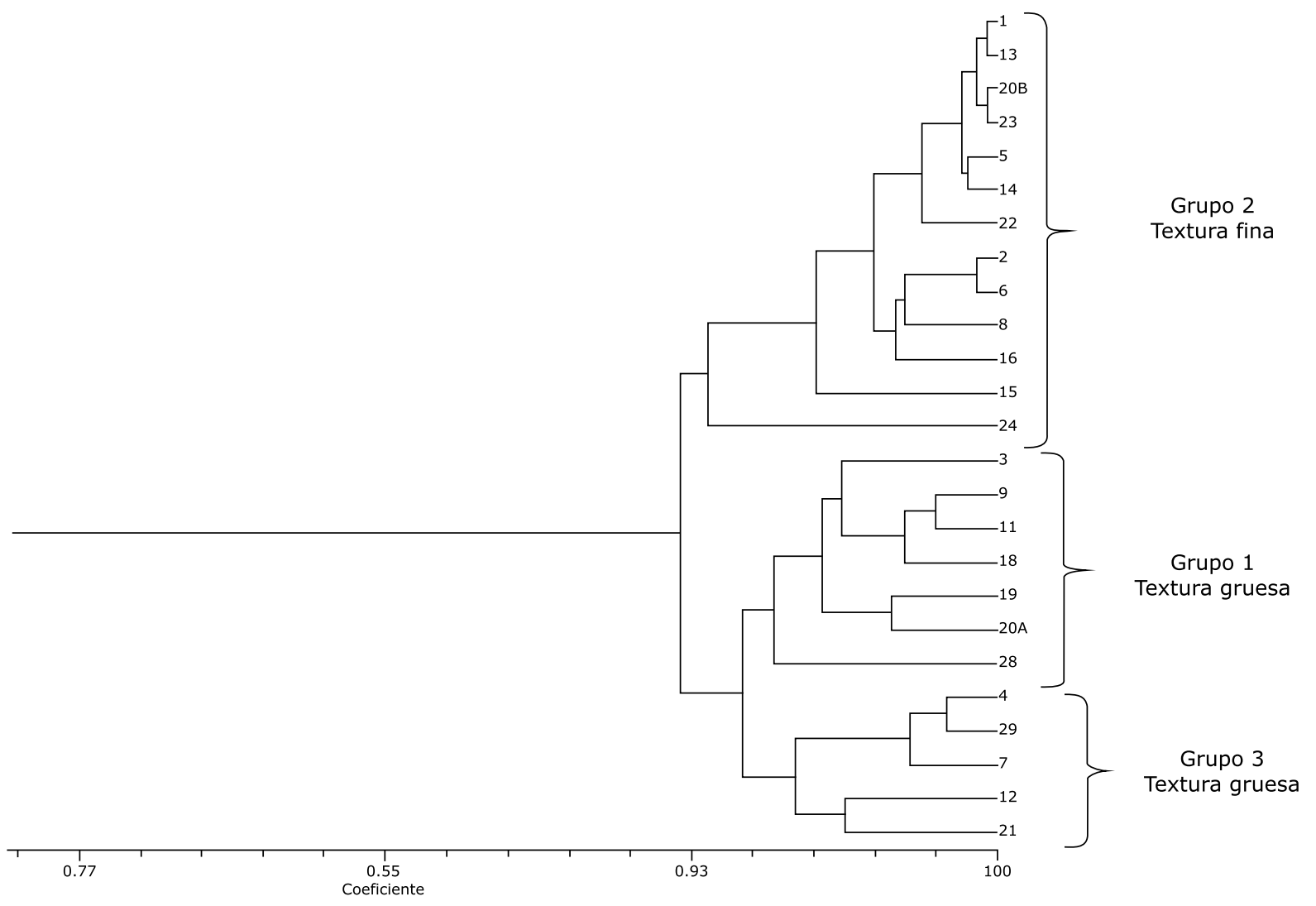

Figura 2. Dendrograma generado de acuerdo con los índices de similaridad utilizando los datos de la combinación de cebadores E-ACC/M-CAG. 


\section{CONCLUSIÓN}

El presente estudio ha permitido el desarrollo de un protocolo para el estudio de caña flecha a nivel molecular. Los resultados permiten concluir que la técnica de AFLPs generó información suficiente para garantizar que los individuos analizados constituyen una colección de diferentes genotipos con fingerprintings únicos, los cuales segregan en forma de tres grupos de acuerdo con su distancia genética corroborando la información previamente obtenida en un estudio de caracterización morfoagronómica.

\section{AGRADECIMIENTOS}

Los autores expresan sus agradecimientos al personal del laboratorio de Biología Molecular Instituto de Investigación de Recursos Biológicos "Alexander von Humboldt" - CIAT y a los funcionarios del Laboratorio de Biotecnología Vegetal de la Universidad de Córdoba, Colombia por su valiosa colaboración.

\section{BIBLIOGRAFÍA}

Aramendiz, H.; Espitia, M. y Robles, J. 2005. Colección, caracterización morfoagronómica y producción de semilla de caña flecha (Gynerium sagittatum AUBL) del Caribe Colombiano. CIUC, Universidad de Córdoba, Montería, p5254.

Ayanog `lu, H.; Bayazit, S.; I'nan, G.; Bakir, M.; Akpinar, A.; Kazan, K. y Ergu"I, A. 2007. AFLP analysis of genetic diversity in Turkish green plum accessions (Prunus cerasifera L.) adapted to the Mediterranean region. Scientia Horticulturae 114:263-267.

Balasaravanan, T.; Pius, P.; Raj Kumar, R.; Muraleedharan, N. y Shasany, A. 2003. Genetic diversity among south Indian tea germplasm (Camellia sinensis, $C$. assamica and C. assamica spp. lasiocalyx) using AFLP markers. Plant Science 165 365-372.

Bassam, B.; Caetano Anolles, G. y Gresshoff, P. 1991. Fast and sensitive silver staining of DNA in polyacrylamide gels. Analytical Biochemistry 196(1):80-83.

Bentolila, S.; Hardy, T.; Guitton, C. y Freyssinet, G. 1992. Comparative genetic analyses of F2 plants and anther culture derived plants of maize. Genome 35:575-582.
Besse, P.; Taylor, G.; Carroll, B.; Berding, N.; Burner, D. y McIntyre, C. 1998. Assessing genetic diversity in a sugarcane germplasm collection using an automated AFLP analysis. Genetica 104(2):143-153.

Bonin, A.; Ehrich., D. y Manel, S. 2007. Statistical analysis of amplified fragment length polymorphism data: a toolbox for molecular ecologists and evolutionists. Molecular Ecology 16:3737-3758.

Chawla, H. 2002. MolecularMarkers and MAS. Introduction to Plant Biotechnology, Science Publishers, Plymouth, p329-358.

Dice, L. 1945. Measures of the amount of ecological association betweem species. Ecology 26:297-302.

Ferreira, M.; Satagopan, J.; Yandel, B.; William, P. y Osborn, T. 1995. Genetic mapping of loci controlling vernalization in rapeseed (Brassica napus L.) Theoretical and Applied Genetics 90:727-732.

Fregene, M.; Bernal, A.; Duque, M.; Dixon, A.; Tohme M. 2000. AFLP analysis of African cassava (Manihot esculenta Crantz) germplasm resistant to the cassava mosaic disease (CMD). Theoretical and Applied Genetics 100(5):678-685. 
Karpa, K.; Bhat K.; Ayad, W. y Hodgkin, T. 1997. Molecular Tools in Plant Genetic Resources Conservation: A Guide to the tecnologies. IPGRI Technical Bulletin No. 2. International Plant Genetic Resources Institute, Rome, p107-117.

Lima, M.; Garcia, A.; Oliveira, S.; Matsuoka, H.; Arizono, C.; De Souza, J. y De Souza, A. 2002. Analysis of genetic similarity detected by AFLP and coeffcient of parentage among genotypes of cane Saccharum spp. Theoretical and Applied Genetics 104:30-38.

Nei, M. y Li, W. 1979. Mathematical model for studying genetic variation in terms of restriction endonucleases. Proceedings of the National Academy of Sciences 76(10):5269-5273.

Newbury, H. y Ford-Lloyd, B. 1993. The use of RAPD for assessing variation in plants. Plant Growth Regulator 12:43-51

Prashanth, S.; Parani, M.; Mohanty, B.; Talame, V.; Tuberosa, R. and Parida, A. 2002. Genetic diversity in cultivars and landraces of Oryza sativa subsp. indica as revealed by AFLP markers. Genome 45:451-459.

Phillips, T.; Murphy, J. y Gooman, M. 1993. Isozyme variation in germplasm accessions of the wild oat Avena sterilis L. Theoretical and Applied Genetics 86:54-64.
QIAGEN. 2004. QIAprep Spin Miniprep Kit. http://www1.qiagen.com/products/plas mid/qiaprepminiprepsystem/qiaprepspin miniprepkit.aspx. [Accedido: 08-122008].

Rohlf, F. (1998). NTSYS-PC, Numerical taxonomy and multivariate analysis system. Version 1.60. Aplied Biostatistics, New York.

Selvi, A.; Nair, N.; Noyer, J.; Singh, N.; Balasundaram, N.; Bansal, K.; Koundal, K. and Mohapatra, T. 2005. Genomic constitution and genetic relationship among the tropical and subtropical Indian sugarcane cultivars revealed by AFLP. Crop Science 45: 1750-1757.

Vos, P.; Hogers, R.; Bleeker, M.; Reijans, M.; Van De Lee, T.; Hornes, M.; Frijters, A.; Pot, J.; Peleman, J.; Kuiper, M. y Zabeau, M. 1995. AFLP: a new technique for DNA fingerprinting. Nucleic Acids Research. 23:4407-4414.

Young, N; Zamir, D; Tanksley, S. 1998. Use of isogenic lines and simultaneous probing to identify DNA markers tightly linked to the Tm-2a gene in tomato. Genetics 120:579585. 